\title{
A Low Cost 3D Vision System for Positioning Welding Mobile Robots using a FPGA protoyping system
}

\author{
I. Ibáñez, M.A. Aguirre, A. Torralba and L.G. Franquelo \\ Escuela Superior de Ingenieros. Universidad de Sevilla \\ Avda. Camino de los Descubrimientos s/n, \\ 41092 Sevilla (SPAIN) \\ aguirre@gte.esi.us.es, torralba@ieee.org, leopoldo@ieee.org
}

\begin{abstract}
The aim of this work is to explore some solutions for artificial vision systems applied to welding autonomous robots. We take advantage from the UNSHADES-1 system, developed in Filiation (blind for evaluation). This system can be used for building a powerful 3D vision system. The system concentrates and process the three images in parallel, producing an accurate value of the position, that can be improved if the relative position of the cameras are well defined.
\end{abstract}

\section{INTRODUCTION}

Autonomous Robots are complete systems that operate inside complex environments without being guided and controlled by human operators. They can establish relationships, take decisions in run-time and adapt their action plans to different external circumstances. The received information make them auto-programmables [1-2], altering their program in order to solve the actual situation. Those system have some AI in their behaviour. Most of cases, the required informations are related to position, speed, acceleration, strength, torque, sizes and shapes from objects and temperature. Quantification of those measures are made by means of mechanical, optical, termical, electrical, ultrasounds, ... sensors. However, advanced research specially takes into account artificial vision transducers. This research can take advantage from the newest microelectronic systems, where design schedule have been shortened using the advanced FPGA's. These devices can process images in "real time", processing frame by frame on the fly.

Between the artificial vision based solutions, the most attractive ones for Robotics applications are object recognition and classification, ensambling, welding, steering and synchronisation with other robots.

One of the most complex application is welding systems based on autonomous robots. An emerging technology that concentrates strategies based on metalurgy, robotics, electrical and electronic engineering and microelectronics. Welding robots are widespread demanded systems, specially where the industry has a high level of automation.

This paper relates a new custom system for determining the exact position of the welding point. The method is based on a system with three low cost cameras that are placed and fixed in the three space axes, and computes the position through the simultaneous processing of the images taken by each camera. Each image is binarised and the welding point is obtained using a programmable binary threshold. So, for each camera, the whole image is reduced to a single point. As the three images are processed simultaneously, every coordinate is computed as part of two of them and then the final coordinate is given as an improved precision number. Using a general purpose system based on FPGA's called UNSHADES [4] we have developed and tested the complete system. Typically this system would be made with a DSP system that would spend many time processing every image in a serial way and wouldn't take advantage from the parallelism in terms of accuracy.

This paper shows how to take advantage from the UNSHADES-1 system. We have developed a full controlled

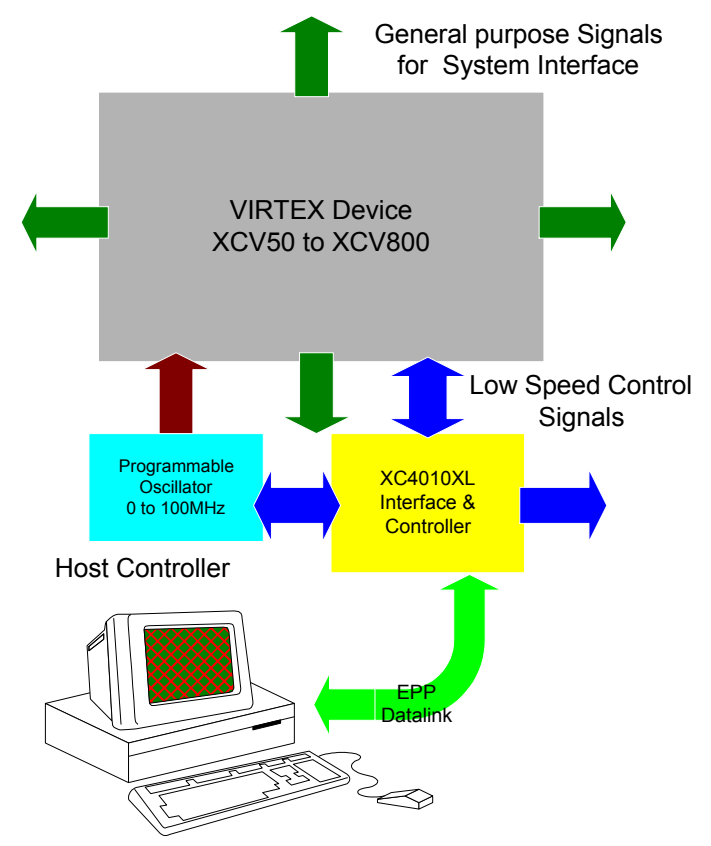

Fig 1. UNSHADES-1 system

system that computes the position of the welding point. In section II we'll describe the UNSHADES framework, in section III the Vision system is presented, section IV shows the system design and finally the conclusions will be presented.

\section{UNSHADES FRAMEWORK}

A Hardware Debugger is a complete programmable system that can be customized for different applications. 
Main advantage is that the complete system can be monitorized during execution time. Main processors are substituted by an advanced FPGA and run embedded into the system itself. If needed a totally customized system can be built using the actual netlist.

UNSHADES-1 general purpose framework that is based on one Xilinx advanced FPGA for emulating the custom processor for, in this case, image processing. Main advantage of UNSHADES system is that the user have full control of the execution of the FPGA due to the special characteristics of this device. UNSHADES means Universidad de Sevilla Hardware Debugger System, and allows a rapid prototyping and the debugging of the processor circuit during execution. Figure 1 shows a general scheme of the system:

Main components of UNSHADES system are listed below:

- $\quad$ An advanced FPGA from Xilinx Virtex family [3]. This FPGA hosts the custom design.

- PC software, the human interface, that provides a comprehensive way of manipulating the inner information of the FPGA.

- A programmable econoscillator from Dallas Semiconductor DS-1075, that generates the system clock. This device can be tuned from the PC.

- There's another FPGA acting as the interface between the PC and the advanced FPGA. All the communications, protocol adapting and signal generation are supported by device, which is smaller than advanced FPGA an has fixed its program.

Communication between PC and UNSHADES hardware is performed using the EPP protocol that can reach 2 Mbytes/s, that is speed enough for our purpose.

UNSHADES software is a complete system that allows a bitstream management including some interesting tools: downloader, internal registers inspector, internal register modifier, oscillator manager and design selector, all fully integrated within the Xilinx standard design flow. For an optimal speed in the bitstream transfer, all the write and read operations are performed using partial reconfiguration techniques.

\section{POSITIONING SYSTEM DESCRIPTION}

The aim of this work is to build a simple, low cost and effective $3 \mathrm{D}$ digital vision system for tracking the position and provide a feedback in positioning autonomous robots. Systems like the one presented can enlarge the effective life of the autonomous robots.

Main drawbacks for constructing these systems are [5]:

$1^{\text {st }}$. Vision systems are as expensive as the robot itself.

$2^{\text {nd }}$. In this kind of robotics systems high throughput techniques that could generate the information in real-time are needed. $3^{\text {rd }}$. There're many problems related with visual information, like shadows, illuminations, uni, bi and three-dimensional, hidden objects, ... etc.

The main objective is to overcome most of these difficulties:

$1^{\text {st }}$ We provide with low cost CCD cameras and video decoders, the cost of the system strongly depends on the required precision. For our prototype we used small digital cameras.

$2^{\text {nd }}$ The processing techniques do not need to process the whole image of each camera. A simplified method allows a resource saving that makes a device like Virtex V300 big enough for the three cameras. They are processed in parallel and the information generated simultaneously, so the result is produced using the same image frame taken in the same instant.

$3^{\text {rd }}$ All the imaging problems are solved due to the use of three cameras placed at different positions. Trinocular stereo vision increases the geometric constraints and reduces the influence of heuristic constraints for stereo matching [6-7]. Most of cases we'll have redundant information, but hidden objects will be for one camera, while the others will continue producing the information. When the information is redundant, some precision improvement techniques can be applied and better weld tracking will be obtained.

$4^{\text {th }}$ Another improvement comes from the image distortion due to the lenses that are commonly curve. For its compensation, a distortion factor is introduced for modifying the typical linear model of the camera. The parameters for the compensation are measured using photogrametric methods for calibration [8-9 ].

Another advantages are introduced when compared with other welding systems: There's no need to produce stimuli for interaction measurement. Our system can be considered a passive system, due to there's no need emitters, because the welding point lighting is the signal taken for measurements.

There're another techniques for providing the information that have been rejected for our purpose: ultrasound are slow and far objects are bad measured with many well known problems. X rays need to use between the object and the sensor an amplifier for transforming $\mathrm{X}$ rays into visible spectrum.

Finally, infrared have many problems related with absorption, many distortions coming from the surrounding emitters.

In our system the three images are mixed, compensated, stored and processed in the same device. 


\section{PROTOTYPE DESCRIPTION}

Our prototype has been designed and implemented in the related FPGA. Our external circuit only needs the video acquiring systems: TVCCD-30M Cameras and the digitizers. Inside the FPGA, the compensations system and coordinate computing blocks are programmed.

Figure 2 presents a block diagram of the PCB board. It's compound by the three digitizers, the Phillips SAA7113H, and another communication features, like a RS232 line, a VGA output and a parallel port.

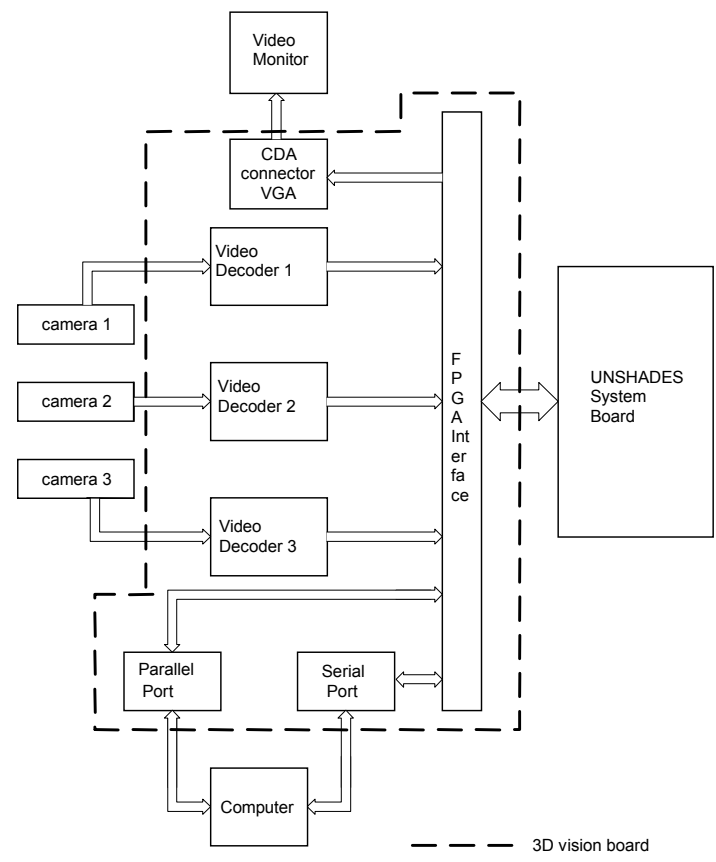

Fig. 2. Main PCB blocks.

Inside the FPGA, a full digital circuit process every image. Figure 3 shows the block diagram for the processor. The first stage is to compensate the lense distortion coming from the camera lenses. Secondly a binarisation of the image searches the welding point, read like a small area obtained that is a small bright circle. After, this part is stored in a memory for post processing.

Previously a circuit configures the digitizers for an adequate image format.

The most important block is the image processor. The first step of the image processing consist of finding the appropiate threshold that would lead to a binarised round shape corresponding to the welding point present in the image. Every pixel is compared with the previously computed threshold, that is obtained from the image condition itself. We have selected the threshold value between the mean of the luminance of the image and its maximum value. If the luminance is below this value the pixel has 0 value (black) and 1 value (white) in another case. The line and pixel number of white values are stored in the memory.

Due to the fixed position of every camera every decoder can produce two values $(\mathrm{x}, \mathrm{y}),(\mathrm{y}, \mathrm{z}) \mathrm{o}(\mathrm{z}, \mathrm{y})$. The video decoders provide information with 625 lines and 1440 pixels per line. This information is useful for generating every coordinate using simple counters.

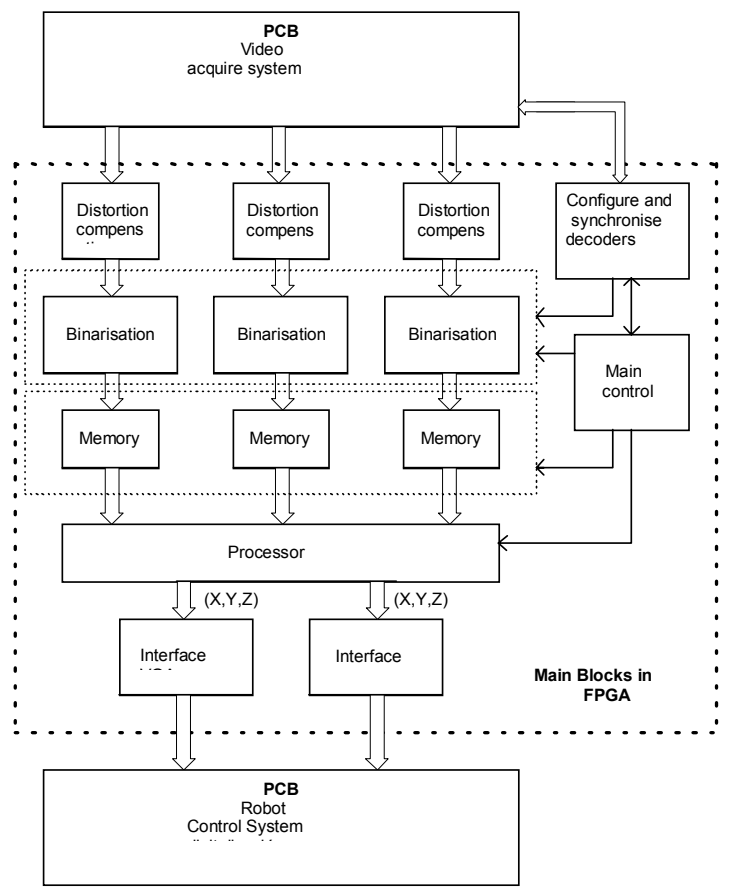

Fig. 3. Inside FPGA processing blocks.

The exact centre of the welding point is computed by means of a gravity centre of each coordinate, using these formulas:

$$
\begin{gathered}
X_{1}=\frac{\sum_{l} x_{i 1 l}^{2}}{\sum_{l} x_{i 1 l}} \quad Y_{1}=\frac{\sum_{m} y_{i 1 m}^{2}}{\sum_{m} y_{i 1 m}} \quad Z_{2}=\frac{\sum_{n} z_{i 2 n}^{2}}{\sum_{n} z_{i 2 n}} \\
X_{3}=\frac{\sum_{p} x_{i 3 p}^{2}}{\sum_{p} x_{i 3 p}} \quad Y_{2}=\frac{\sum_{q} y_{i 2 q}^{2}}{\sum_{q} y_{i 2 q}} \quad Z_{3}=\frac{\sum_{r} z_{i 3 r}^{2}}{\sum_{r} z_{i 3 r}}
\end{gathered}
$$

We'll obtain two numbers for each coordinate because they're repeated in two memories. Some precision improvements can be produced if the cameras are placed with a half pixel of deviation for every coordinate.

$$
X=\frac{X_{1}+X_{3}}{2} \quad Y=\frac{Y_{1}+Y_{2}}{2} \quad Z=\frac{Z_{2}+Z_{3}}{2}
$$


The results are transferred to the PC that provides the information for the controllers. The implementation of this system refresh the coordinates every two frames, due to the post-processing datapath. The complete system has been implemented into a Virtex 300, with a $60 \%$ of the occupation. System clock runs at $50 \mathrm{MHz}$.

\section{CONCLUSIONS}

The paper presents a simple system for determine the absolute position of a welding point of an autonomous robots. Main advantages are the cost, the precision and the computing speed. In the final version of this paper the computing time will be given. It's been estimated like a coordinate computing per two images, which can be considered in terms of robot speed real-time. This system takes advantage of the UNSHADES- 1 system that provides a complete, flexible and very comfortable platform for the vision system.

\section{REFERENCES}

[1] D. Rdiner Hundsdörfer "Costuras perfectas. Aumenta el empleo de la soldadura por láser en el mecanizado de chapa". Revista de Soldadura y Tecnología de unión. Año XII- Num 72, pp. 9-11 Noviembre/Dic 2001.

[2] P. Coiffet, M. Chirouze, "An Introduction to Robot Technology", Mc Graw Hill, 1983.

[3] Xilinx Data Book. 2002

[4] M.A. Aguirre, J. Tombs, A. Torralba, L.G. Franquelo "HADES-1: A rapid prototyping environment based on aadvances FPGA's". Proceedings of the Design of Circuits and Integrated Systems, DCSI'01 Oporto 2001.

[5] J. M. Angulo Usategui "Robótica Práctica. Tecnología y aplicaciones". Capítulo 7: "Visión de Máquinas", Paraninfo, 5. a ed., 2000.

[6] Ayache, "Trinocular Stereo Vision for Robotics",IEEE Trans. Pattern Anal. \& Machine Intelligence, vol. 13, no. 1, Jan. 1991.

[7] Yachida, "3D Data Acquisition by Multiple View", Robotics Research: Third Int. Symp., Faugeras, O.D. and Giralt, G., Eds. Cambridge, MA, MIT Press, pp. 11$18,1986$.

[8] Brown D., 1971: Close-Range Camera Calibration. Photogrammetric Engineering, Vol. 37, No.8

[9] El-Hakim, S.F., 1986: Real-Time Image Metrology with CCD Cameras. Photogrammetric Engineering and Remote Sensing, Vol. 52, No 11, pp. 1757-1766. 